\title{
Clinical utility gene card for: Usher syndrome
}

\author{
Hanno J Bolz ${ }^{\star, 1,2}$ and Anne-Françoise Roux ${ }^{3}$ \\ European Journal of Human Genetics (2011) 19, doi:10.1038/ejhg.2011.15; published online 9 March 2011
}

\section{DISEASE CHARACTERISTICS}

\subsection{Name of the disease (synonyms)}

Usher syndrome (USH). Clinically, USH presents in three clinical subtypes, namely USH1, USH2 and USH3. This categorization is still useful. However, there is considerable variability within a subtype and as a result, overlaps exist between USH1, -2 and -3 . Locus names consist of the clinical subtype and an additional letter that indicates a specific locus, according to the order in which these loci have been mapped. $1,2,3$

\subsection{OMIM\# of the disease}

276900, 276904, 601067, 602083, 606943, 276901, 605472, 611383, 276902.

1.3 Name of the analysed genes or DNA/chromosome segments Genes implicated in Usher syndrome type 1: USH1C, MYO7A, CDH23, PCDH15 and USH1G.

Genes implicated in Usher syndrome type 2: USH2A, GPR98, DFNB31.

Gene implicated in Usher syndrome type 3: CLRN1.

Gene implicated in digenic Usher syndrome type 2 with GPR98 and effective as a modifier of USH2A: PDZD7.

\subsection{OMIM\# of the gene(s)}

Genes implicated in USH1: MYO7A (USH1B): MIM\# 276903; USH1C: MIM\# 605242; CDH23 (USH1D): MIM\# 605516; USH1E: MIM\# 602097 (gene to be identified); PCDH15 (USH1F): MIM\# 605514; USH1G: MIM\# 607696; USH1H: MIM\# 612632 (gene to be identified). Genes implicated in USH2: USH2A: MIM\# 608400; GPR98 (USH2C): MIM\# 602851; DFNB31 (USH2D): MIM\# 607928.

Gene implicated in USH3: CLRN1: MIM\# 606397.

Gene implicated in digenic Usher syndrome and effective as a modifier of USH2A: PDZD7: MIM\# 612971.

The loci USH1A and USH2B have been withdrawn.

\subsection{Mutational spectrum}

Reported mutations are mainly point mutations (missense, nonsense, splicing mutations), but also small deletions, duplications and insertions. Large deletions and duplications have also been described and have become more accessible through diagnostic techniques such as MLPA and array-CGH. Variants identified in each Usher gene are continuously registered in LOVD-USHbases. ${ }^{4}$

1.6 Analytical methods

Several strategies can be used and have different detection rates.
(1) Complete Sanger sequencing of coding exons and flanking intronic sequences.

(2) For USH1: Initial haplotype analysis can help in preselecting the most likely causative gene. If haplotyping is not an option (lack of additional samples from the family, uninformative constellation), genes should be sequenced in the order of their causal frequency: MYO7A, CDH23, PCDH15, USH1C and USH1G. CLRN1 should be considered if all USH1 genes are negative.

(3) For USH2: As in USH1, initial haplotype analysis can help in preselecting the most likely causative gene. The USH2A gene is causative in $70-80 \%$ of cases. In cases where linkage analysis is not an option, direct sequencing of USH2A is the reasonable first step. If this turns out to be negative, the six N-terminal DFNB31 (USH2D) exons should be sequenced, then GPR98 (USH2C). CLRN1 should be considered if all USH2 genes are negative.

(4) Because founder mutations exist in various populations for several USH genes (USH1C, PCDH15, USH2A, CLRN1), the ethnicity of the patient should be taken into account.

(5) In some patients $(<10 \%)$, it can be necessary to look for large rearrangements. This can be performed by array CGH with customized chips for Usher genes. An MLPA kit is available for $P C D H 15$ and is under development for USH2A.

(6) Genotyping microarrays with allele-specific oligonucleotides corresponding to known Usher syndrome-associated sequence variants can be used for simultaneous mutation screening in all USH genes. ${ }^{5}$ The approach is comparatively inexpensive, but because many mutations are private, negative screening does not exclude a causative role for the genes represented on the chip.

(7) Next-generation sequencing (NGS) will soon allow for largescale sequencing of all USH genes in a patient. Although most labs do not offer this type of analysis yet, it will likely become the standard diagnostic approach in the near future.

\subsection{Analytical validation}

Confirmation of a mutation should be performed by de novo amplification and sequencing from the patient's sample. Segregation analysis within a family is highly recommended to ascertain the parental origin of each identified variant. Special care is required for interpretation if variants of unknown clinical significance are identified, such as missense and translationally silent substitutions (exonic synonymous changes and intronic variations).

\footnotetext{
${ }^{1}$ Bioscientia Center for Human Genetics, Ingelheim, Germany; ${ }^{2}$ Institute for Human Genetics, University Hospital of Cologne, Cologne, Germany; ${ }^{3}$ Laboratoire de Génétique Moléculaire, CHU Montpellier, INSERM U827, IURC, Montpellier, France

${ }^{*}$ Correspondence: Dr HJ Bolz, Bioscientia Center for Human Genetics, Konrad-Adenauer-Str. 17, 55218 Ingelheim, Germany or Institute for Human Genetics, University Hospital of Cologne, Kerpener Str. 34, 50931 Cologne, Germany. Tel: +49 6132781 206; Fax: +49 6132781 298; E-mail: hanno.bolz@bioscientia.de
} 
1.8 Estimated frequency of the disease (incidence at birth ('birth prevalence') or population prevalence) The frequency has been reported to be $1 / 25000$ in the United States and Scandinavia. ${ }^{6}$ Following new data, the prevalence may be 5 times higher. $^{7}$

1.9 If applicable, prevalence in the ethnic group of the investigated person

Population- or ethnicity-specific prevalences exist for different subtypes because of founder mutations in certain populations: This is the case for USH3 in Finland and among Ashkenazi Jews, where this subtype accounts for more than $40 \%$ of the cases.

Some mutations are particularly frequent in specific populations (eg, p.Arg1502X/CDH23 in Swedes; p.Arg245X/PCDH15 in Ashkenazi Jews; c.216G > A/USH1C in Acadians and Quebecois; p.Tyr176X/ CLRN1 in Finns (see LOVD-USHbases)). ${ }^{4}$

\subsection{Diagnostic setting}

\begin{tabular}{lcc}
\hline & Yes & No \\
A. (Differential) diagnostics & $\bigotimes$ & $\square$ \\
B. Predictive testing & $\square$ & $\square$ \\
C. Risk assessment in relatives & $\bigotimes$ & $\square$ \\
D. Prenatal & $\bigotimes$ & $\square$
\end{tabular}

\section{Comment:}

Prenatal diagnosis is rarely requested. Because the hearing deficit can be compensated by hearing aids or, in case of USH1 and sometimes USH3, cochlear implants, requests for prenatal diagnosis should be discussed in detail in genetic counseling. Prenatal diagnosis remains exceptional.

\section{TEST CHARACTERISTICS}

\begin{tabular}{|c|c|c|c|c|}
\hline & \multicolumn{2}{|c|}{ Genotype or disease } & \multirow{2}{*}{$\begin{array}{l}\text { A: True positives } \\
\text { B: False positives }\end{array}$} & \multirow{2}{*}{$\begin{array}{l}\text { C: False negative } \\
\text { D: True negative }\end{array}$} \\
\hline & Present & Absent & & \\
\hline \multicolumn{5}{|l|}{ Test } \\
\hline \multirow[t]{2}{*}{ Positive } & $A$ & $\mathrm{~B}$ & Sensitivity: & $A /(A+C)$ \\
\hline & & & Specificity: & $\mathrm{D} /(\mathrm{D}+\mathrm{B})$ \\
\hline \multirow[t]{2}{*}{ Negative } & C & $\mathrm{D}$ & Positive predictive value: & $A /(A+B)$ \\
\hline & & & Negative predictive value: & $D /(C+D)$ \\
\hline
\end{tabular}

\subsection{Analytical sensitivity}

(proportion of positive tests if the genotype is present)

More than $90 \%$ for USH1.

Around $80 \%$ for USH 2 .

USH3: probably more than $90 \%$.

\subsection{Analytical specificity}

(proportion of negative tests if the genotype is not present) $100 \%$.

\subsection{Clinical sensitivity}

(proportion of positive tests if the disease is present)

The clinical sensitivity can be dependent on variable factors such as age or family history. In such cases a general statement should be given, even if a quantification can only be made case by case.
Because of extensive genetic heterogeneity, genetic testing is rarely carried out for all known USH genes in patients who lack mutations in the major genes (this limitation will probably be overcome with the introduction of NGS into molecular genetic testing). If comprehensive analysis was carried out for all known exons, the clinical sensitivity would probably be $\sim 80 \%$ for USH1 and USH 2 .

CLRN1 mutations can cause an USH1- or USH2-like phenotype, and USH3 individuals may therefore sometimes not be subjected to CLRN1 testing. Estimation of the clinical sensitivity for USH3 is therefore difficult.

\subsection{Clinical specificity}

(proportion of negative tests if the disease is not present)

The clinical specificity can be dependent on variable factors such as age or family history. In such cases a general statement should be given, even if a quantification can only be made case by case.

Almost $100 \%$.

\subsection{Positive clinical predictive value}

(lifetime risk to develop the disease if the test is positive)

$100 \%$ if two clearly pathogenic alleles have been identified. The phenotype can vary even within families. Moreover, mutations in most USH genes can also result in either allelic non-syndromic phenotype (recessive deafness in case of missense mutations in MYO7A, USH1C, CDH23, PCDH15, DFNB31, dominant hearing loss in case of MYO7A, non-syndromic RP in case of USH2A). Especially for MYO7A (USH1B), the genotype-phenotype correlation is not clear-cut: Some missense changes may cause non-syndromic deafness while others result in additional retinal dystrophy, making prediction of retinal affliction in young children with previously undescribed missense mutations a challenge.

\subsection{Negative clinical predictive value} (probability not to develop the disease if the test is negative) Assume an increased risk based on family history for a non-affected person. Allelic and locus heterogeneity may need to be considered.

Index case in that family had been tested: Not applicable.

Index case in that family had not been tested: Not applicable.

\section{CLINICAL UTILITY}

3.1 (Differential) diagnosis: The tested person is clinically affected (To be answered if in 1.10 'A' was marked)

\subsubsection{Can a diagnosis be made other than through a genetic test?}

\begin{tabular}{|c|c|c|}
\hline No & $\square$ (continue with 3.1.4) & \\
\hline \multirow[t]{7}{*}{ Yes } & $\nabla$ & \\
\hline & Clinically & $\nabla$ \\
\hline & Imaging & $\square$ \\
\hline & Endoscopy & $\square$ \\
\hline & Biochemistry & $\square$ \\
\hline & Electrophysiology & $\square$ \\
\hline & Other (please describe) & $\otimes$ \\
\hline
\end{tabular}

Note: A negative genetic test does not exclude the clinical diagnosis because of genetic heterogeneity. 
3.1.2 Describe the burden of alternative diagnostic methods to the patient

Regular investigation of hearing and visual function remains important even after having confirmed the diagnosis genetically in order to provide appropriate support to the patient. Electrophysiological tests such as electroretinogram (ERG) and dark adaptation tests are time-consuming and stressful.

In children born with congenital deafness, several clinical investigations are recommended in order to exclude the presence of a syndrome: ECG (to detect Jervell and Lange-Nielsen syndrome that can implicate life-threatening cardiac arrhythmias or SANDD syndrome ${ }^{8}$ ), ERG and eye fundoscopy, thyroid function (Pendred syndrome), renal function (Alport syndrome). Genetic testing can help adapt the clinical surveillance (clearly, pathogenic mutations in an Usher gene would justify regular follow-up by ophthalmologists and audiologists, but the other investigations mentioned above would become dispensable). This would unburden the patient, but also save costs for the health-care system.

3.1.3 How is the cost effectiveness of alternative diagnostic methods to be judged?

In deaf children with an up to $10 \%$ probability of developing additional retinal degeneration because of Usher syndrome, Sanger sequencing of the major genes for the different Usher subtypes is laborious, but probably less expensive than regular clinical follow-ups by several different disciplines (see Section 3.1.2). With high-throughput simultaneous genotyping, for example, by next-generation sequencing, entering the field of genetic diagnostics, the genetic approach will certainly pay off.

3.1.4 Will disease management be influenced by the result of a genetic test?

\begin{tabular}{|c|c|c|}
\hline No & $\square$ & \\
\hline \multirow[t]{4}{*}{ Yes } & $凶$ & \\
\hline & $\begin{array}{l}\text { Therapy } \\
\text { (please describe) }\end{array}$ & $\begin{array}{l}\text { There is not yet a therapy for Usher syndrome, but } \\
\text { there are rehabilitation strategies: Hearing aids are } \\
\text { an important support in USH2 and USH3. Bilateral } \\
\text { cochlear implant can compensate for the hearing } \\
\text { deficit in USH1 and advanced USH3. }\end{array}$ \\
\hline & $\begin{array}{l}\text { Prognosis } \\
\text { (please describe) }\end{array}$ & $\begin{array}{l}\text { Precise prognosis regarding the progression of } \\
\text { hearing loss (in USH2 and USH3) and retinal } \\
\text { disease (for all subtypes) remains difficult } \\
\text { because even intrafamilial variability can often be } \\
\text { observed. }\end{array}$ \\
\hline & $\begin{array}{l}\text { Management } \\
\text { (please describe) }\end{array}$ & Management of hearing impairment (see 3.1.4). \\
\hline
\end{tabular}

3.2 Predictive setting: The tested person is clinically unaffected but carries an increased risk based on family history

(To be answered if in 1.10 ' $\mathrm{B}$ ' was marked)

\subsubsection{Will the result of a genetic test influence lifestyle and prevention?}

If the test result is positive (please describe):

Yes. The audiological and visual handicaps reduce the patient's mobility and narrow the choices regarding professions.

If the test result is negative (please describe):

If extensive early genetic testing in a hearing-impaired person has been carried out retinal degeneration in later life is unlikely, making choice of professions that require intact vision an option.
3.2.2 Which options in view of lifestyle and prevention does a person at risk have if no genetic test has been done (please describe)?

All options if hearing is normal. If hearing is congenitally abnormal, Usher syndrome is likely (depending on the genetic distance to the index case) and the restrictions are as said under Section 3.2.1.

3.3 Genetic risk assessment in family members of a diseased person (To be answered if in 1.10 ' $\mathrm{C}$ ' was marked)

\subsubsection{Does the result of a genetic test resolve the genetic situation in that family? \\ Yes.}

3.3.2 Can a genetic test in the index patient save genetic or other tests in family members?

No. However, (a) normal hearing would make the diagnosis very unlikely and genetic testing unnecessary, (b) congenital hearing impairment could be due to other causes (environmental or genetic, eg, GJB2 mutations), (c) co-occurrence of deafness and retinal degeneration in a close relative would make the presence of the same mutations as in the index patient very likely.

\subsubsection{Does a positive genetic test result in the index patient enable a} predictive test in a family member?

Yes.

\subsection{Prenatal diagnosis}

(To be answered if in 1.10 ' $\mathrm{D}$ ' was marked)

Possible when two confirmed deleterious mutations are identified in the family. Rarely requested (see Section 1.10).

3.4.1 Does a positive genetic test result in the index patient enable a prenatal diagnosis?

This depends on the carrier status of the partner. Because numerous variants of unknown clinical significance can be identified in the Usher genes, genetic counseling can be complicated.

Also, the possibility of digenic inheritance should be considered (as has been shown for $C D H 23 / P C D H 15^{9}$ and GPR98/PDZD $7^{10}$ ). Moreover, alleles in second loci may act as modifiers (as has been shown for heterozygous MYO7A alleles modifying USH3 $\mathrm{A}^{11}$ and heterozygous $P D Z D 7^{10}$ alleles modifying USH2A).

\section{IF APPLICABLE, FURTHER CONSEQUENCES OF TESTING}

Please assume that the result of a genetic test has no immediate medical consequences. Is there any evidence that a genetic test is nevertheless useful for the patient or his/her relatives? (Please describe)

For many patients, the knowledge about the genetic defect is valuable in itself. In particular, patients with nonsense mutations could benefit from future translational read-through therapy approaches. $^{12}$

Knowledge of the responsible gene and its mutations may give access to future therapies. Moreover, the identification of a mutation excludes differential diagnoses (see Section 3.1)

Parents of children with USH1 may consider training their children in vision-independent modes of communication such as tactile signing.

\section{CONFLICT OF INTEREST}

HJB may appear to have a conflict of interest because he currently works at the Bioscientia Center for Human Genetics, which is part of a publicly traded diagnostic company. He is actively engaged in research and teaching at the University Hospital of Cologne. HJB affirms that 
the entire body of research is unrelated to his employment at Bioscientia, was not sponsored by Bioscientia, and has no bearing on the research or clinical programs at Bioscientia. A-FR declares no conflict of interest.

\section{ACKNOWLEDGEMENTS}

This work was supported by EuroGentest, an EU-FP6-supported NoE, contract number 512148 (EuroGentest Unit 3: 'Clinical genetics, community genetics and public health', Workpackage 3.2).

1 Keats BJB, Lentz J (Updated 29 June 2010). Usher Syndrome Type I; in GeneReviews at GeneTests: Medical Genetics Information Resource (database online). Copyright, University of Washington, Seattle, WA, 1997-2010. Available at http://www.genetests. org (accessed 26 September 2010).

2 Keats BJB, Lentz J (Updated 14 April 2010). Usher Syndrome Type II; in GeneReviews at GeneTests: Medical Genetics Information Resource (database online). Copyright, University of Washington, Seattle, WA, 1997-2010. Available at http://www.genetests. org (accessed 26 September 2010).
3 Saihan Z, Webster AR, Luxon L, Bitner-Glindzicz M: Update on Usher syndrome. Curr Opin Neurol 2009; 22: 19-27.

4 URL: LOVD-USHbases: https://grenada.lumc.nl/LOVD2/Usher_montpellier/.

5 Cremers FP, Kimberling WJ, Külm M et al: Development of a genotyping microarray for Usher syndrome. J Med Genet 2007; 44: 153-160.

6 Boughman JA, Fishman GA: A genetic analysis of retinitis pigmentosa. Br J Ophthalmol 1983; 67: 449-454.

7 Kimberling WJ, Hildebrand MS, Shearer AE et al: Frequency of Usher syndrome in two pediatric populations: implications for genetic screening of deaf and hard of hearing children. Genet Med 2010; 12: 512-516.

8 Baig SM, Koschak A, Lieb A et al: Loss of $\mathrm{Ca}_{\mathrm{v}} 1.3$ (CACNA1D) function in a human channelopathy with bradycardia and congenital deafness. Nat Neurosci 2011; 14: 77-84.

9 Zheng QY, Yan D, Ouyang XM et al: Digenic inheritance of deafness caused by mutations in genes encoding cadherin 23 and protocadherin 15 in mice and humans. Hum Mol Genet 2005; 14: 103-111.

10 Ebermann I, Phillips JB, Liebau MC et al: PDZD7 is a modifier of retinal disease and a contributor to digenic Usher syndrome. J Clin Invest 2010; 120: 1812-1823.

11 Adato A, Kalinski $\mathrm{H}$, Weil D, Chaib H, Korostishevsky M, Bonne-Tamir B: Possible interaction between USH1B and USH3 gene products as implied by apparent digenic deafness inheritance. Am J Hum Genet 1999; 65: 261-265.

12 Welch EM, Barton ER, Zhuo J et al. PTC124 targets genetic disorders caused by nonsense mutations. Nature 2007; 447: 87-91. 